\title{
Mathematical modeling of the geometrical differences between the weld end crater and the steady-state weld pool
}

Cite as: J. Laser Appl. 32, 022024 (2020); https://doi.org/10.2351/7.0000068

Submitted: 01 April 2020. Accepted: 01 April 2020 . Published Online: 28 April 2020

Antoni Artinov (D), Victor Karkhin, Marcel Bachmann (D), and Michael Rethmeier (iD)

\section{COLLECTIONS}

Paper published as part of the special topic on Proceedings of the International Congress of Applications of Lasers \& Electro-Optics (ICALEO<sup>\&\#174; </sup> 2019)

Note: This paper is part of the Special Collection: Proceedings of the International Congress of Applications of Lasers

(B)

\& Electro-Optics (ICALEO 2019).
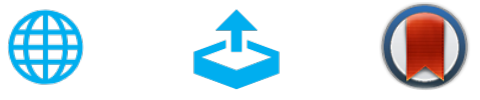

\section{ARTICLES YOU MAY BE INTERESTED IN}

Theoretical study of influence of electromagnetic stirring on transport phenomena in wire feed laser beam welding

Journal of Laser Applications 32, 022026 (2020); https://doi.org/10.2351/7.0000069

Image-based algorithm for nozzle adhesion detection in powder-fed directed-energy deposition

Journal of Laser Applications 32, 022021 (2020); https://doi.org/10.2351/7.0000070

Spattering effects during selective laser melting

Journal of Laser Applications 32, 022023 (2020); https://doi.org/10.2351/7.0000061

\section{Sellight Highlights of the best new research} in the physical sciences 


\title{
Mathematical modeling of the geometrical differences between the weld end crater and the steady-state weld pool
}

Cite as: J. Laser Appl. 32, 022024 (2020); doi: 10.2351/7.0000068

Submitted: 1 April 2020 - Accepted: 1 April 2020 .

Published Online: 28 April 2020

Antoni Artinov, ${ }^{7}$ (D) Victor Karkhin, ${ }^{2}$ Marcel Bachmann, ${ }^{2}$ (D) and Michael Rethmeier ${ }^{1,2,3}$ (iD)

\begin{abstract}
AFFILIATIONS
${ }^{1}$ BAM Federal Institute for Materials Research and Testing, Unter den Eichen 87, Berlin 12205, Germany

${ }^{2}$ Department of Welding and Laser Technologies, Peter the Great St. Petersburg Polytechnic University, Polytechnicheskaya str. 29, 195251 St. Petersburg, Russia

${ }^{3}$ Department of Joining Technology, Berlin Institute of Technology, 10623 Berlin, Germany
\end{abstract}

Note: This paper is part of the Special Collection: Proceedings of the International Congress of Applications of Lasers \& ElectroOptics (ICALEO ${ }^{\circledR}$ 2019).

\begin{abstract}
The geometrical characteristics of the weld end crater are commonly used as a means of validating numerical results in welding simulations. In this paper, an analytical model is developed for calculating the cooling stage of the welding process after the moving energy source is turned off. Solutions for various combinations of heat sources and heated bodies are found. It is shown that after turning off the energy source, additional melting of the base material in the longitudinal direction may occur due to the overheated liquid metal. The developed technique is applied to complete-penetration keyhole laser beam welding of $2 \mathrm{~mm}$ thick austenitic stainless-steel plate $316 \mathrm{~L}$ at a welding speed of $20 \mathrm{~mm} \mathrm{~s}^{-1}$ and a laser power of $2.3 \mathrm{~kW}$. The results show a theoretical increase in the weld end crater length of up to $19 \%$ compared to the length of the steady-state weld pool. It is found that at the moment of switch off, the weld end crater center, where solidification of the liquid metal ends, is shifted from the heat source axis toward the weld pool tail. The solidification rate and the direction of crystallization of the molten material during the welding process and those in the weld end crater differ significantly. A good agreement between the computational results and the welding experiments is achieved.
\end{abstract}

Key words: Weld end crater, Steady-state weld pool, Mathematical modeling, Solidification, Laser beam welding

Published under license by Laser Institute of America. https://doi.org/10.2351/7.0000068

\section{INTRODUCTION}

The weld end crater zone differs from the rest of the weld seam by the solidification rate and the direction of crystallization. It is a zone of increased irregularity of chemical composition, increased transient and residual stresses, and increased susceptibility to hot and cold cracking. ${ }^{1-3}$ From a geometric point of view, the weld end crater is a depression in the weld face at the termination of the weld bead. ${ }^{4}$

For verification of the calculated weld pool dimensions, the experimental dimensions of the weld end crater are often taken up.

This raises the following questions: (1) Do the shape and the dimensions of the weld pool and the weld end crater correspond so well to each other? (2) Does the axis of the heat source coincides with the center of the end crater when it is switched off? From the physical point of view, these questions can be formulated as follows: (1) Does the base material melt after the heat source is switched off? (2) Does the maximum temperature zone shift during crystallization of the molten material at the end of the process? Thermal processes in the weld pool, the weld metal, and the heat affected zone are analyzed in many publications. ${ }^{3,5-10}$ Nevertheless, the thermal behavior of the weld end crater remains not sufficiently studied.

The aim of the present study is to develop an analytical method for determining the size of the weld end crater and the solidification kinetics of its liquid metal. Based on an example of 
laser beam welding of thin steel plates, the significant geometric difference between the weld end crater and the weld pool should be shown

\section{THEORY}

In order to calculate the formation of the weld end crater and its final characteristics, the information of the steady-state temperature field and the temperature equalization period after the heat source is switched off should be known. Hence, at first, the temperature fields and, consequently, the sizes of the weld end crater and the molten pool for two combinations of a moving heat source and a welded body are compared: (1) a point heat source and a slab (the temperature field is three-dimensional) and (2) a uniformly distributed line heat source and a thin plate (the temperature field is two-dimensional). Hereby, the following assumptions are made:

- The heat source of a constant power $q$ moves along the $x$-axis with a constant speed $v$ long enough to achieve a quasistationary state (the temperature field does not change in the moving coordinate system $x, y, z$ related to the heat source) [Figs. 1(a) and 1(b)]. Then, the source is instantly switched off, leading to geometrical changes of the weld pool up to the moment of complete solidification of the liquid metal;

- the body with a thickness $h$ is homogeneous and infinitely wide and long [Fig. 1(a)];

- the material properties (thermal conductivity $\lambda$, thermal diffusivity $a$, and volume-specific heat capacity $c \rho$ ) are temperatureindependent;

- the surface heat transfer coefficients $\alpha_{1}$ and $\alpha_{2}$ do not depend on temperature; and

- the initial body temperature and the room temperature $T_{0}$ are constant.

The point heat source on the surface of a slab with insulated surfaces is considered first [Fig. 1(a)]. The heat source is turned off at point $O$. This moment is taken as initial $(t=0)$. Further absence of a real heat source is represented as a simultaneous action of two combined fictitious sources: a positive source of power $+q$, which is a continuation of the real heat source, and a negative source (sink) of power $-q$ [Fig. 1(c)]. Hence, the temperature field after switching off the real energy source in the moving coordinate system $x, y, z$, associated with the sources, will be an algebraic sum of the steady-state

a)

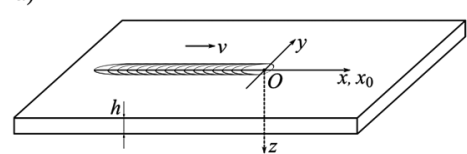

c)

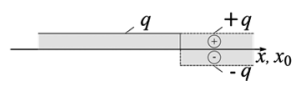

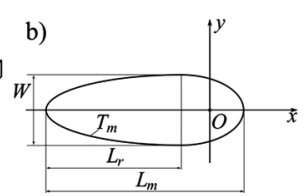

FIG. 1. Schematic for the calculation of the heat conduction process after the heat source is switched off: (a) coordinate system; (b) weld pool dimensions (melting isotherm $T_{m}$; weld pool length $L_{m}$; length of the tail part of the weld pool $L_{r}$ ); and $(c)$ the mathematical model for the heat source. temperature field due to the fictitious heat source and the unsteady contribution due to the fictitious heat sink:

$$
\begin{aligned}
T(x, y, z, t)-T_{0}= & {\left[1-\Psi_{3}(x, y, z, t)\right] \frac{q}{2 \pi \lambda} } \\
& \times \exp \left(\frac{-v x}{2 a}\right) \sum_{i=-\infty}^{\infty} \frac{1}{R_{i}} \exp \left(\frac{-v R_{i}}{2 a}\right),
\end{aligned}
$$

with $R_{i}=R \sqrt{1+4 i\left(\frac{h}{R}\right)^{2}\left(i-\frac{z}{h}\right)}$ and $R=\sqrt{x^{2}+y^{2}+z^{2}}$.

The heat saturation function $\Psi_{3}$ is defined as the fraction of time-dependent temperature distribution and the limit quasistationary state of the temperature field (theoretically at $t=\infty$ ) in a moving coordinate system. Here, the heat saturation function is represented as

$$
\begin{gathered}
\Psi_{3}(x, y, z, t)=\left[\sum _ { i = - \infty } ^ { \infty } \frac { 1 } { \rho _ { 3 i } } \operatorname { e x p } ( - \rho _ { 3 i } ) \frac { 1 } { 2 } \left[\Phi^{*}\left(\frac{\rho_{3 i}}{2 \sqrt{\tau_{3}}}-\sqrt{\tau_{3}}\right)\right.\right. \\
\left.\left.+\exp \left(2 \rho_{3 i}\right) \Phi^{*}\left(\frac{\rho_{3 i}}{2 \sqrt{\tau_{3}}}+\sqrt{\tau_{3}}\right)\right]\right] / \sum_{i=-\infty}^{\infty} \frac{1}{\rho_{3 i}} \exp \left(-\rho_{3 i}\right),
\end{gathered}
$$

where $\rho_{3 i}=\rho_{3} \sqrt{1+4 i\left(\frac{\delta}{\rho_{3}}\right)\left(i-\frac{\zeta}{\delta}\right)} ; \quad \rho_{3}=\frac{v R}{2 a} ; \quad \delta=\frac{v h}{2 a} ; \zeta=\frac{v z}{2 a} ; \quad$ and $\Phi^{*}(u)=\operatorname{erfc}(u)=1-\frac{2}{\sqrt{\pi}} \int_{0}^{\mathrm{u}} \exp \left(-x^{2}\right) \mathrm{d} x$.

The complimentary error function $\Phi^{*}$ is included in all computer systems. For a point heat source, the function $\psi_{3}$ on the upper $(\zeta=0)$ and on the lower $(\zeta=\delta)$ surfaces of a slab with dimensionless thicknesses $\delta=1$ and $\delta=4$ is shown in Figs. 2(a) and 2(b). It depends on dimensionless time $\tau_{3}$ and dimensionless distance $\rho_{3}$ (distance from the observation point to the center of the coordinate system). It can be seen that the closer to the heat source (the smaller the distance $\rho_{3}$ ), the earlier the temperature reaches its limit value. On the lower surface $\left(\zeta=\delta\right.$ and $\rho_{3} \geq \delta$ ), the values of $\Psi_{3}$ are slightly higher (dashed curves) than that on the upper surface $(\zeta=0$, solid curves). If the lower surface of the slab $(z=h)$ does not affect the temperature field (theoretically, for $h=\infty$ ), Eqs. (1) and (2) are simplified since only the members of the series at $i=0$ remain,

$$
\begin{aligned}
T(x, y, z, t)-T_{0}= & {\left[1-\Psi_{3}(x, y, z, t)\right] } \\
& \times \frac{q}{2 \pi \lambda R} \exp \left(\frac{-v(x+R)}{2 a}\right),
\end{aligned}
$$

$$
\begin{aligned}
\text { where } \Psi_{3}(x, y, z, t)=\frac{1}{2}[ & \Phi^{*}\left(\frac{\rho_{3}}{2 \sqrt{\tau_{3}}}-\sqrt{\tau_{3}}\right) \\
& \left.+\exp \left(2 \rho_{3}\right) \Phi^{*}\left(\frac{\rho_{3}}{2 \sqrt{\tau_{3}}}+\sqrt{\tau_{3}}\right)\right] .
\end{aligned}
$$

The function $\Psi_{3}$ is shown in Fig. 2(c). By comparing Figs. 2(a), 2(b), and 2(c), it can be seen that with the growth of 
a)

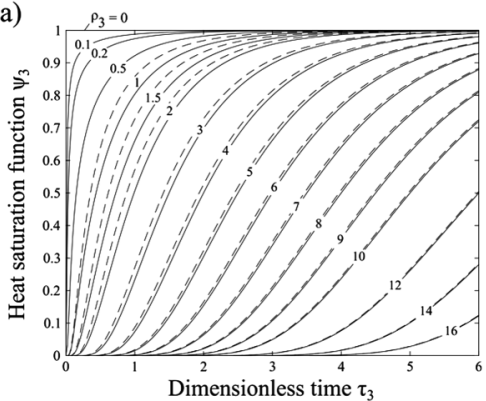

b)

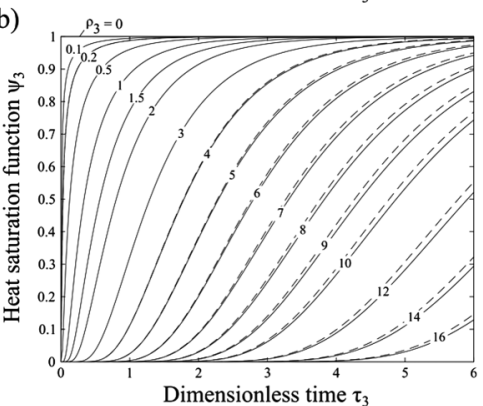

c)

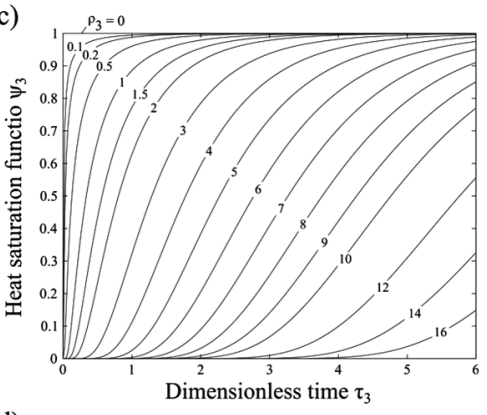

d)

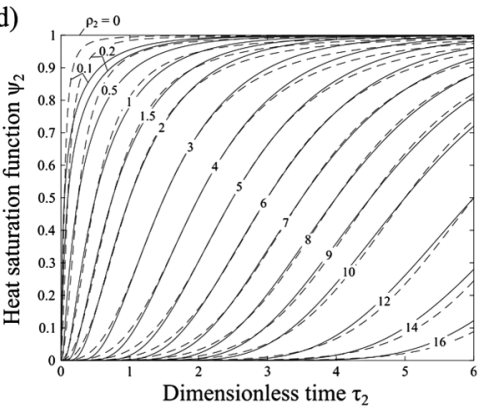

FIG. 2. Heat saturation functions for a point heat source on the surface of a slab of dimensionless thickness $\delta,\left(\Psi_{3}\right)$, and a line source in a thin plate $\left(\Psi_{2}\right)$, depending on the dimensionless time $\left(\tau_{2}\right.$ and $\left.\tau_{3}\right)$ and dimensionless distance $\left(\rho_{2}\right.$ and $\left.\rho_{3}\right)$ : (a) for the upper (solid curves) and lower (dashed curves) surface of the slab, $\delta=1$; (b) for the upper (solid curves) and lower (dashed curves) surface of the slab, $\delta=4$; (c) for the surface of a massive body, $\delta=\infty$; and (d) for the plate.

dimensionless thickness $\delta$, the values of the function $\Psi_{3}$ at $\tau=$ const slightly increase.

As next, the uniformly distributed line heat source in a thin plate is considered. Here, the temperature field is two-dimensional, which means that there is no temperature drop over the thickness of the plate. The algorithm of solving the corresponding heat conduction problem is similar to the considered algorithm for a point heat source on a slab. The temperature field after termination of the line heat source in the moving coordinate systems $x, y$, associated with the heat source [Fig. 1(a)], is described by the following equation:

$$
\begin{aligned}
T(x, y, t)-T_{0}=[1 & \left.-\Psi_{2}(x, y, t)\right] \\
& \times \frac{q / h}{2 \pi \lambda} \exp \left(\frac{-v x}{2 a}\right) \mathrm{K}_{0}\left(\frac{v r}{2 a} \sqrt{1+\frac{4 a b}{v^{2}}}\right),
\end{aligned}
$$

with $r=\sqrt{x^{2}+y^{2}}$ and $b=\frac{\alpha_{1}+\alpha_{2}}{c \rho h}$.

Here, $\mathrm{K}_{0}$ is the modified Bessel function of second kind of zero order, $r$ is the distance in a plane from the center of the origin, $b$ is the coefficient of heat loss for a thin plate, and $\alpha_{1}$ and $\alpha_{2}$ are the surface heat transfer coefficients on the top and bottom of the plate. The heat saturation function $\Psi_{2}$ is calculated as follows: ${ }^{8,9}$

$$
\begin{aligned}
\Psi_{2}(x, y, t)= & \frac{1}{2 \mathrm{~K}_{0}\left(\rho_{2}\right)} \\
& \quad \times \int_{0}^{2 \tau_{2} / \rho_{2}} \frac{1}{u} \exp \left(\frac{-\rho_{2}}{2}\left(u+\frac{1}{u}\right)\right) \mathrm{d} u,
\end{aligned}
$$

where $\rho_{2}=\frac{v r}{2 a} \sqrt{1+\frac{4 a b}{v^{2}}}$ and $\tau_{2}=\frac{v^{2} t}{4 a}\left(1+\frac{4 a b}{v^{2}}\right)$.

The function $\Psi_{2}$ is shown in Fig. 2(d) (solid lines). Since the integral in (6) is not expressed as known functions, it is convenient to use an approximation formula: $:^{8,9}$

$$
\Psi_{2}(x, y, t)=\left[1+\left(\frac{\rho_{2}}{2 \tau_{2}}\right)^{1.7+0.4 \rho_{2}}\right]^{-1} .
$$

The curves calculated by Eq. (7) are shown in Fig. 2(d), highlighted with dashed lines. The approximation error is significant only for small values of $\rho_{2}$. The approximation function (7) coincides with the exact function in the limiting cases: $\Psi_{2}\left(\rho_{2}, 0\right)=0$, $\Psi_{2}\left(\rho_{2}, \infty\right)=1$, and $\Psi_{2}\left(\infty, \tau_{2}\right)=0$.

Thus, it is possible to calculate the temperature field and estimate the size of the weld end crater using Eqs. (1)-(7).

The effect of boundedness of the welded body can be taken into account by using the method of images. ${ }^{6-9}$

Note that the curves $\Psi_{2}$ and $\Psi_{3}$ allow estimating the time of action of a moving source $t$ (or the initial length of the weld seam $v t$ ), necessary to achieve a quasistationary state of the temperature field (for example, the steady-state characteristics of the weld seam) with a given relative accuracy. ${ }^{6-9}$

Similarly, by making use of the method of sources (Green's function method), it is possible to obtain solutions to the heat conduction problems for concentrated sources shifted relative to the origin of coordinates. If a distributed heat source is used, it can be represented as a sum of concentrated sources. In this case, the solution to the heat conduction problem results from the integration of the power distribution of the distributed source. , $^{8,9}$ 


\section{EXAMPLE}

A plate of austenitic chrome-nickel steel $316 \mathrm{~L}(0.03 \% \mathrm{C}, 17 \%$ $\mathrm{Cr}, 10 \% \mathrm{Ni}, \mathrm{Fe}$ bal.) with a thickness of $2 \mathrm{~mm}$ is welded using the keyhole technique. The process occurs under the following conditions: laser beam power of $q_{\text {gross }}=2.3 \mathrm{~kW}$ and welding speed of $v=20 \mathrm{~mm} \mathrm{~s}^{-1}$. The experimental weld seam width $W$ on the upper surface lies in the range between 1.56 and $1.79 \mathrm{~mm}$ (Fig. 3). The experimental length $L_{c}$ and width $W_{c}$ of the weld end crater are in the range of $4.19-4.79 \mathrm{~mm}$ and $1.58-1.81 \mathrm{~mm}$, respectively.

The temperature difference over the thickness of the plate is negligibly small (the difference in the weld width between the upper and lower surfaces is less than 3\%), so the scheme of a uniformly distributed line heat source in the plate is used to calculate the temperature field. The following input data are taken: $\lambda=0.032 \mathrm{~W} \mathrm{~mm}^{-1} \mathrm{~K}^{-1}$, $\alpha_{1}=\alpha_{2}=0, \quad a=6.4 \mathrm{~mm}^{2} \mathrm{~s}^{-1}, c \rho=0.005 \mathrm{~J} \mathrm{~mm}^{-3} \mathrm{~K}^{-1}, T_{0}=293 \mathrm{~K}$, and $T_{m}=1773 \mathrm{~K}$.

The effective (net) power of the laser beam $q$ is unknown, as the absorption coefficient $\eta_{h}$ is highly dependent on many factors. As a result of calibration of the numerical model based on the sizes of the weld pool and the weld end crater, the net power value $q=1085 \mathrm{~W}$ and the efficiency $\eta_{h}=q / q_{\text {gross }}=0.47$ are obtained. The temperature field after the laser beam is turned off $(t \geq 0)$ is calculated by using Eqs. (5) and (7). The transition from the moving coordinate system $x, y, z$, connected with the energy source, to the fixed coordinate system $x_{0}, y, z$, with the origin at point $O$, is realized by the equation: $x=x_{0}-v t$, where $t$ is the time after switching off the heat source.

At the moment of termination of the laser beam, the isotherm $T_{m}$ describes the boundary of the weld pool in a quasistationary state [curve $t=0$ in Fig. 4(b)]. In the beginning (at $t<0.043 \mathrm{~s}$ ), the base material continues to melt in front of the molten pool due to overheating of the liquid metal. The area of the new molten metal is darkened in the figure. At the same time, the tail part of the weld pool solidifies. Thereafter (at $t<0.043 \mathrm{~s}$ ), the liquid metal starts solidifying from all sides. The zone of the maximum temperature in the molten metal shifts from point $O$ (the heat source axis at the time of shutdown) to point $A$, where solidification of the liquid a)

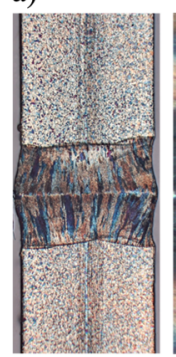

b)

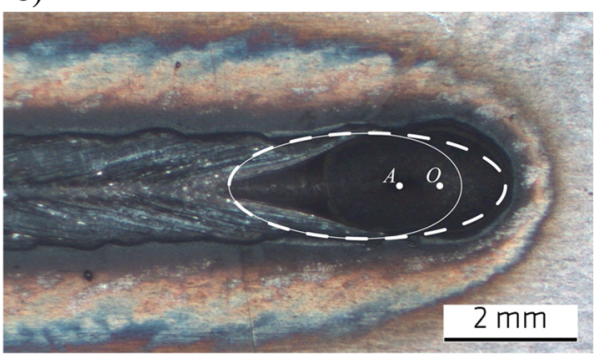

FIG. 3. Laser beam welded plate of $316 \mathrm{~L}$ chrome-nickel austenitic steel with thickness of $2 \mathrm{~mm}$ (laser beam power of $2300 \mathrm{~W}$, welding speed of $20 \mathrm{~mm} \mathrm{~s}^{-1}$ ): (a) the metallographic cross section; (b) top view of the weld and the weld end crater. The calculated contours of the weld pool (solid lines) and the weld end crater (dashed lines) are highlighted in white. Point $A$ shows the position, where the solidification of the liquid metal in the weld end crater ends. Point $O$ shows the origin of the heat source axis at the time of shutdown. a)

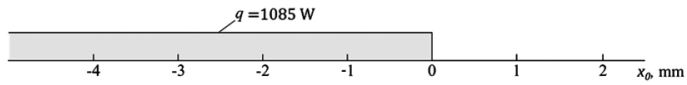

b)

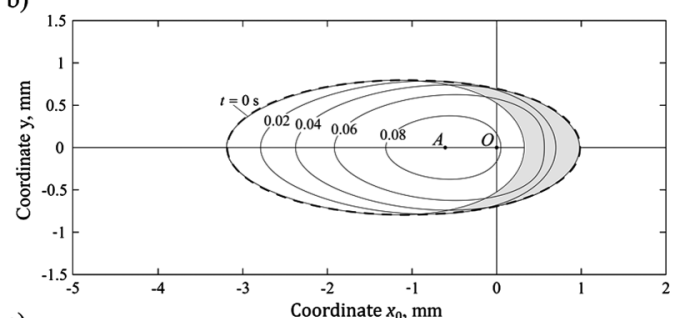

c)

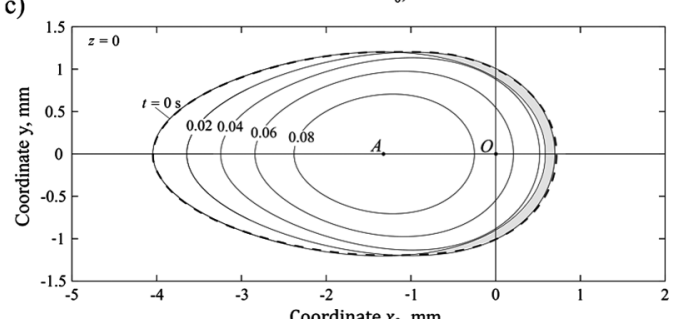

d)

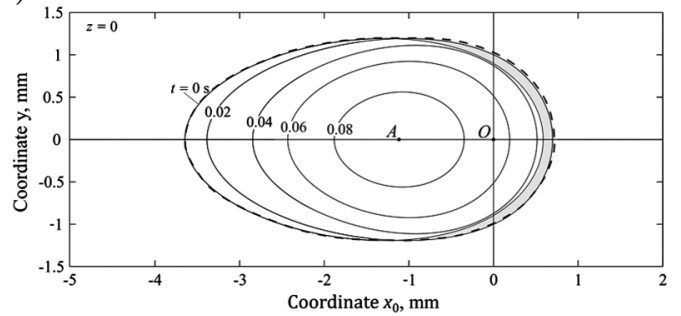

FIG. 4. Formation of the weld end crater (highlighted by a thick dashed line) after the heat source is switched off: (a) effective power distribution $q$ along the weld axis; (b) time dependence of the position of the melting isotherm $T_{m}$ for a line heat source in a thin plate of thickness $h=2 \mathrm{~mm}$; (c) time dependence of the position of the melting isotherm $T_{m}$ for a point heat source on a slab of thickness $h=2 \mathrm{~mm}$; and (d) time dependence of the position of the melting isotherm $T_{m}$ for a point heat source on a massive body, $h=\infty$.

metal in the weld end crater ends [Fig. 4(b)]. Typically, on welded samples, this point (the center of the end crater) is visible as the deepest point on the end crater surface. Note that when heated by a moving distributed heat source (e.g., a normally distributed or uniformly distributed circular source), the maximum temperature zone is also shifted backward from the heat source axis. ${ }^{8,9}$

The end crater area includes all the molten metals after the source is switched off. The boundary of the weld end crater is shown with a bold dashed line [Fig. 4(b)]. It can be seen that the width of the end crater $W_{c}$ coincides with the width of the weld pool in the steady-state $\left(W_{c}=W\right)$, but the length of the weld end crater $L_{c}$ is much greater than the length of the weld pool $L_{m}$ (about $0.66 \mathrm{~mm}$ or $19 \%$ ). This means, strictly speaking, that the size of the weld end crater cannot be considered as the size of the weld pool for heat source calibration purposes.

This geometrical difference between the weld end crater and the steady-state weld pool leads to metallurgical changes, e.g., increased 
irregularity of the chemical composition. Consequently, in combination with other physical effects, e.g., increased transient and residual stresses, the weld end crater represents a zone with high susceptibility to different welding defects, such as hot and cold cracking.

It is well known that the growth rate and the shape of the crystal axes are mainly determined by the speed of movement and the shape of the weld pool interface (melting isotherm $T_{m}$ ). Since the velocities and the shapes of the isotherm $T_{m}$ in the weld end crater and the molten pool are significantly different [Fig. 4(b)], the growth velocities and trajectories of the crystals should differ significantly. In this case, the maximum solidification rate of the weld end crater is greater than the maximum solidification rate of the weld pool, which is equal to the welding speed.

For comparison with the experiment in Fig. 3, the calculated boundary of the weld pool at the moment of switching off the laser beam in the form of a closed thin white line and the calculated boundary of the weld end crater highlighted by a thick dashed line are shown. The computed position of the center of the end crater (point $A$ ) is adjusted to the experimental one. It can be seen that the calculated dimensions of the tail part of the molten pool (width $W$ and length $L_{r}$ ) satisfactorily coincide with the experimental values. The experimental straight-line shape of the weld pool differs from the computed rounded one, which is explained primarily by the effect of latent heat of solidification and the high welding speed. ${ }^{8,9}$ The calculated dimensions of the weld end crater boundary lie in the observed experimental range. Thus, in general, there is a qualitative agreement between the results of the calculation and the experiment.

Naturally, the question arises: Will the base metal melts after switching off the heat source with other combinations of sources and heated body types? To prove this, an additional combination of a point source on the surface of a $2 \mathrm{~mm}$ thick slab with the same welding parameters is considered $\left(q=1085 \mathrm{~W}, v=20 \mathrm{~mm} \mathrm{~s}^{-1}\right)$. In such combination, the temperature field is three-dimensional. As it can be seen from Fig. 4(c), the geometric difference between the weld end crater and the weld pool is qualitatively the same as for the line heat source and the thin plate [Fig. 4(b)]. In the case of a point heat source on a slab [Fig. 4(c)], the width and the length of the weld end crater on the top surface are greater than in the thin plate [Fig. 4(b)]. The offset of the center of the end crater (point $A$ ) from the axis of the laser beam (point $O$ ) is also bigger. However, in this case, the calculated maximum temperature on the lower surface does not reach the melting point.

As a limiting case, the combination of a point heat source on a semi-infinite body is considered, see Fig. 4(d). The comparison of Fig. 4(d) with Fig. 4(c) shows that the width and the length of the weld end crater are smaller for the semi-infinite body. Nevertheless, the volume of the metal melted after the laser beam is turned off is approximately the same.

According to the obtained results, for the different combinations of heat sources and heated bodies, the width and the length of the tail part of the weld end crater and the weld pool are the same. However, the total length of the end crater can be significantly longer (up to 19\%) than the length of the molten pool. Therefore, in the general case, it is not perfectly correct to consider the size of the weld end crater to be equal to the size of the steady-state weld pool. The center of the end crater, where the solidification of the liquid metal ends, is shifted toward its tail relative to the axis of the heat source at the time of termination of its action. A similar process of solidification is observed in arc welding of aluminum alloy with thickness of 3 and $5 \mathrm{~mm} .^{1,3}$

It should be emphasized that an additional consideration of temperature-dependent material properties, different heat source distributions, and other physical factors will only lead to quantitative changes in the geometric differences between the weld end crater and the steady-state weld pool, whereby qualitative differences will remain.

\section{CONCLUSIONS}

- The developed analytical method for solving the problem of heat conduction allows to calculate temperature fields and all their characteristics after termination of the heat source.

- After the termination of the heat source, additional melting of the base metal due to an overheated liquid metal in the weld pool is possible. Therefore, in the general case, the size of the weld end crater cannot be considered to be equal to the size of the steady-state molten pool. The developed technique is applied to complete-penetration keyhole laser beam welding of a $2 \mathrm{~mm}$ thick austenitic chrome-nickel 316L steel plate at a welding speed of $20 \mathrm{~mm} \mathrm{~s}^{-1}$ and a laser power of $2.3 \mathrm{~kW}$. The results show a theoretical increase in the weld end crater length in comparison to the length of the steady-state weld pool by up to $19 \%$.

- At the moment of switch off, the weld end crater center, where solidification of the liquid metal ends, is shifted from the heat source axis toward the weld pool tail.

- The solidification rate and the solidification direction of the liquid metal in the steady-state weld pool and those in the weld end crater differ significantly.

- The negligible heat transfer in thickness direction during thin sheets welding leads to the biggest geometrical and metallurgical differences. These, in combination with the typical for the laser beam welding high transient and residual stresses, can increase the susceptibility to hot and cold cracking.

\section{ACKNOWLEDGMENTS}

Financial funding from the Deutsche Forschungs-gemeinschaft (DFG, German Research Foundation) Project No. 411393804 is gratefully acknowledged. The authors sincerely thank N. Bakir and A. Gumenyuk for the execution of experiments.

\section{NOMENCLATURE}

a

$b$

$c \rho$

$h$

$\mathrm{K}_{0}$

$L_{c}$

$L_{m}$

$L_{r}$

$q$

qgross 


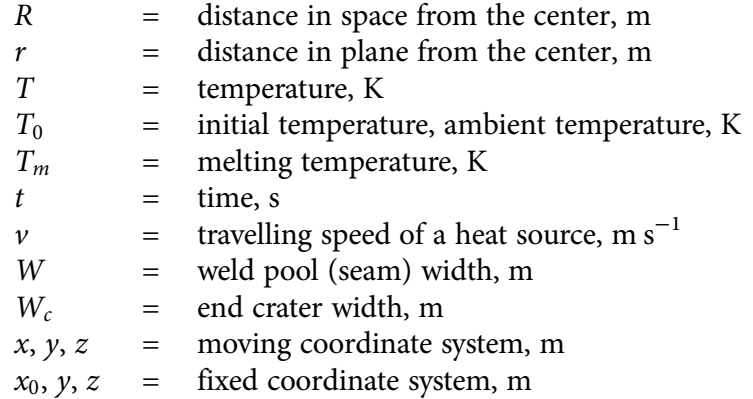

\section{Greek}

$\begin{array}{ll}\alpha_{1} \text { and } \alpha_{2} & =\text { heat transfer coefficients on the top and bottom } \\ \delta & =\text { dimfaces, } \mathrm{W} \mathrm{m}^{-2} \mathrm{~K}^{-1} \\ \eta_{h} & =\text { heat source efficiency } \\ \lambda & =\text { thermal conductivity, } \mathrm{W} \mathrm{m}^{-1} \mathrm{~K}^{-1} \\ \zeta & =\text { dimensionless coordinate over thickness } \\ \rho_{2} & =\text { dimensionless distance in plane from the center } \\ \rho_{3} & =\text { dimensionless distance in space from the center } \\ \tau & =\text { dimensionless time } \\ \Phi^{*} & =\text { complementary error function } \\ \Psi & =\text { heat saturation function }\end{array}$

\section{REFERENCES}

${ }^{1}$ N. N. Prokhorov, Technological Strength of Welds During Crystallisation (Metallurgiya, Moscow, 1979), p. 248 (in Russian).

${ }^{2}$ E. L. Makarov and B. F. Yakushin, Theory of Weldability of Steels and Alloys (Moscow State Technical University, Moscow, 2014), p. 487 (in Russian).

${ }^{3} \mathrm{H}$. Guo, J. Hu, and H. L. Tsai, "Formation of weld crater in GMAW of aluminum alloys," Int. J. Heat Mass Transfer 59, 5533-5546 (2009).

${ }^{4}$ ANSI/AWS A3.0, Standard Welding Terms and Definitions.

${ }^{5}$ D. Rosenthal, "The theory of moving sources of heat and its application to metal treatments," Trans. ASME 68, 849-865 (1946).

${ }^{6}$ N. N. Rykalin, Thermal Principles in Welding. Part 1. Heat Flow in Arc Welding (USSR Academy of Sciences, Moscow, 1947), p. 271 (in Russian).

${ }^{7}$ N. N. Rykalin, "Calculation of heat flow in welding," translated by Z. Paley and C. M. Adams, Jr., Contract No. UC-19-066-001-C3817 (Moscow, 1951), p. 337.

${ }^{8}$ V. A. Karkhin, Thermal Processes in Welding, 2nd ed. (St. Petersburg Polytechnic University, Publishing, St. Petersburg, 2015), p. 572.
${ }^{9}$ V. A. Karkhin, Thermal Processes in Welding (Springer, Singapore, 2019), p. 515 .

${ }^{10}$ A. Artinov, M. Bachmann, and M. Rethmeier, "Equivalent heat source approach in a 3D transient heat transfer simulation of full-penetration high power laser beam welding of thick metal plates," Int. J. Heat Mass Transfer 122, 1003-1013 (2018).

\section{Meet the Authors}

Antoni Artinov, born in 1991 in Aytos (Bulgaria), received his M.Sc. in the field of Engineering Science from the Berlin Institute of Technology and his M.Sc. in Mechanical Engineering from the Tomsk Polytechnic University. Since 2016, he is working as a research assistant at the Federal Institute for Materials Research and Testing in Berlin, Germany, in the department "Welding Technology." Currently, he is working in the field of numerical simulations in laser beam welding processes.

Professor Dr.-Ing. Victor Karkhin works at the Department of Welding and Laser Technologies of the Peter the Great St. Petersburg Polytechnic University. He received his diploma and Ph.D. degree at the Peter the Great St. Petersburg Polytechnic University. His experience includes mathematical modelling of thermal, metallurgical, and mechanical processes in fusion welding.

Dr.-Ing. Marcel Bachmann, born 1984 in Berlin, is with the BAM Federal Institute for Materials Research and Testing in Berlin, Germany, in the department "Welding Technology" since 2009 where he is leading the working group "Welding Simulation." He received his diploma from the Berlin Institute of Technology in Engineering Science and his Ph.D. for numerical investigations of electromagnetically assisted high power laser beam welding processes. Currently, he is working on several projects involving numerical simulations in welding processes.

Professor Dr.-Ing. Michael Rethmeier is with the BAM Federal Institute for Materials Research and Testing. He is the head of the division "Welding Technology." $\mathrm{He}$ is also heading the "Chair of Joining Technology" at the Institute of Machine Tools and Factory Management, Berlin Institute of Technology and is division director of "Joining and Coating Technology" at the Fraunhofer Institute for Production Systems and Design Technology. Present research topics include among others innovative arc welding processes, high power laser beam welding, and numerical simulations in various welding processes. 